\title{
Hyaluronic acid injections in post-enucleation or evisceration socket syndrome: a case series
}

\author{
Renata Migliardi ${ }^{1}$ Alessandra Modugno ${ }^{2} \cdot$ Fabrizio Chirico $^{3} \cdot$ Nicola Zerbinati $^{4} \cdot$ Giovanni Francesco Nicoletti $^{5}$. \\ Giorgio Lo Giudice ${ }^{6}$ (1) Raffaele Rauso ${ }^{3}$
}

Received: 14 April 2021 / Accepted: 6 July 2021 / Published online: 11 August 2021

(C) The Author(s) 2021, corrected publication 2021

\begin{abstract}
Background One of the primary goals of enucleation and evisceration surgery is the restoration of an adequate orbital volume through the use of appropriately sized alloplastic or autogenous tissues. In patients inadequately treated, post-enucleation or evisceration socket syndrome occurs. Fillers are an ideal alternative for eyelid and eyebrow arcade volume enhancement since their injection is easily performed in an outpatient setting avoiding several complications. The aim of this study is to report the use of hyaluronic acid (HA) fillers to treat volume deficits of the upper and lower eyelids, projecting the brow arcade and reducing the asymmetry.

Methods Thirteen patients ( 2 male, 11 female, mean age 32.7 years) were treated from June 2012 to May 2020. Non-surgical treatment by HA filler injection for aesthetic rehabilitation of deep superior sulcus, inferior tear trough deformity, and scleral show was performed.

Results No complications as orbital-ache and/or vasovagal response were reported during the injections. Minor complications such as light swelling at the site of injection, self-resolved within 2 days, were recorded. Stable results were observed at follow-ups. In two cases, two successive treatments were required at 3 and 6 years from the first injection.

Conclusions Hyaluronic acid fillers offer a versatile and safe method for replacing soft tissue lost from the upper eyelid/brow complex in cases of post-enucleation or evisceration socket syndrome.

Level of evidence: Level IV, therapeutic study.
\end{abstract}

Keywords Hyaluronic acid $\cdot$ Post-enucleation socket syndrome $\cdot$ Post evisceration socket syndrome $\cdot$ PESS $\cdot$ Filler

Giorgio Lo Giudice

giorgio.logiudice@gmail.com

Turin, Italy

2 Ocularistica Italiana, Via Duilio, 13, 00192 Roma, Italy

3 Multidisciplinary Department of Medical-Surgical and Dental Specialties, Oral and Maxillofacial Surgery Unit, University of Campania "Luigi Vanvitelli", Via Luigi De Crecchio, 6, 80138 Naples, Italy

4 Dermatology Department, University of Insubria, Via Guicciardini, 9, 21100 Varese, Italy

5 Multidisciplinary Department of Medical-Surgical and Dental Specialties, Plastic Surgery Unit, University of Campania "Luigi Vanvitelli”, Via Luigi De Crecchio, 6, 80138 Naples, Italy

6 Department of Neurosciences, Reproductive and Odontostomatological Sciences, Maxillofacial Surgery Unit, University of Naples "Federico II", Via Pansini, 5, 80131 Naples, Italy

\section{Introduction}

The loss of an eye has a huge disheartening impact on the social life of a patient. It significantly affects the individual's physical, psychological, emotional, and social well-being. The aim of the surgery (the enucleation for malignant tumors or the evisceration for trauma or other causes) is to replace the orbital volume and to allow the use of a prosthetic eye [1-3]. For those patients whose aesthetic outcomes become especially important, depending on the cause of the eye loss, one of the primary goals of enucleation and evisceration surgery is the restoration of an adequate orbital volume through the use of appropriately sized alloplastic or autogenous tissues [4].

In patients inadequately treated, the post-enucleation or evisceration socket syndrome (PESS), or superior sulcus syndrome, occurs. PESS is characterized by enophthalmos, sulcus deformity, upper and lower lid malposition, and 
backward tilt of the prosthesis [5]. In such instances, the volume replacement aims to create a sustainable palpebral aperture symmetry, skin crease, sulcus fullness, and minimal motility of the implants, thus allowing a correct prosthesis fit, partial mobility, and good closure of the eyelids. Unfortunately, even when the orbital volume is correctly restored, postoperative fat atrophy is a common condition that leads to a superior sulcus deformity on the upper eyelid, and sometimes a tear trough deformity on the lower eyelid, therefore, causing aesthetic concerns [6].

In the age of non-surgical facial rejuvenation, injectable fillers are considered a less invasive volume replacement option that avoids the morbidity of additional orbital or eyelid surgeries. Fillers are an ideal alternative for the eyelid and eyebrow arcade volume enhancement since their injection is easily performed in an outpatient setting, avoiding general anesthesia, and offering a high degree of tolerability and satisfaction [7]. Hyaluronic acid (HA) fillers are injectable gels commonly used in aesthetic facial rejuvenation. The dynamic viscosity that makes them unlikely to migrate from their placement and the quick and repeatable procedure, that can be performed under topical or local anesthesia, make it a suitable treatment for volume deficit restorations [8].

This study aims to report the use of HA fillers in treating volume deficits of the upper and lower eyelids, and to project the brow arcade to reduce the asymmetry with the normal contralateral eye.

\section{Materials and methods}

In these retrospective case series, 13 patients ( 2 male, 11 female, mean age 32.7 years) were treated from June 2012 to May 2020. A senior ophthalmologist (RM) injected all patients treated, and the technique remained consistent throughout the years. The full written informed consent was obtained. Eight patients were previously treated with eye enucleation, and underwent a dermal-fat graft ( 3 of them after a secondary implant extrusion), 5 patients were previously treated with eye evisceration. Every patient complained about a significant upper eyelid sulcus, and 4 patients also complained about inferior scleral show. Nonsurgical treatment by HA filler injection for the aesthetic rehabilitation of the undesirable deep superior sulcus, inferior tear trough deformity, and scleral show was performed.

A local anesthetic was not required. To reduce the upper sulcus and to restore the projection of the sub-ciliary crease and eyebrow, the HA filler was injected in a supra-periosteal layer at the superior orbital rim, and above the levator palpebrae superioris muscle at the supra-tarsal crease. Two different HA fillers, with different rheology, were used per each layer; in both layers, $1 \mathrm{~mL}$ of HA gel was injected. In the supra-periosteal layer, a $28 \mathrm{mg} / \mathrm{mL}$ HA gel filler with a G' value of 420-480 Pa (Neauvia Intense, Matex Lab Spa, Brindisi, Italy) was used above the levator palpebrae superioris muscle; in the supra-tarsal crease, a $22 \mathrm{mg} / \mathrm{mL}$ with 270-320 G' value filler (Neauvia Rheology, Matex Lab Spa, Brindisi, Italy) was used. Each $\mathrm{mL}$ of HA filler was distributed using 2 to 3 retrograde releases by 27 Gauge canula. No post-treatment restrictions were necessary and the patient was discharged under the outpatient protocol. The pre-treatment, post-treatment, and follow-up documentation were achieved using photographs measurements.

\section{Results}

No complications as orbital-ache and/or vasovagal response were reported during the injections. Minor complications such as slight swelling at the site of injection, self-resolved within 2 days, were recorded in almost all cases. In one case, due to a superficial injection in the supra-tarsal crease, 20 $\mathrm{U}$ of hyaluronidase was injected to solve an excessive bulging appearance. All the patients were happy with the results achieved, both in static and dynamic features (Figs. 1, 2, and 3).

Stable results were observed at follow-ups with no need for further injections in 11 patients. In two cases, two successive treatments were required after 3 and 6 years from the first injection (Fig. 4).

\section{Discussion}

The major challenge in craniofacial reconstructive surgery of the orbital area is to correct congenital or acquired deformities. Anophthalmic sockets can exhibit progressive
Fig. 1 Patient 1 open eyelid view. Pre-treatment (A), immediate post-treatment (B), and 1-year follow-up (C)

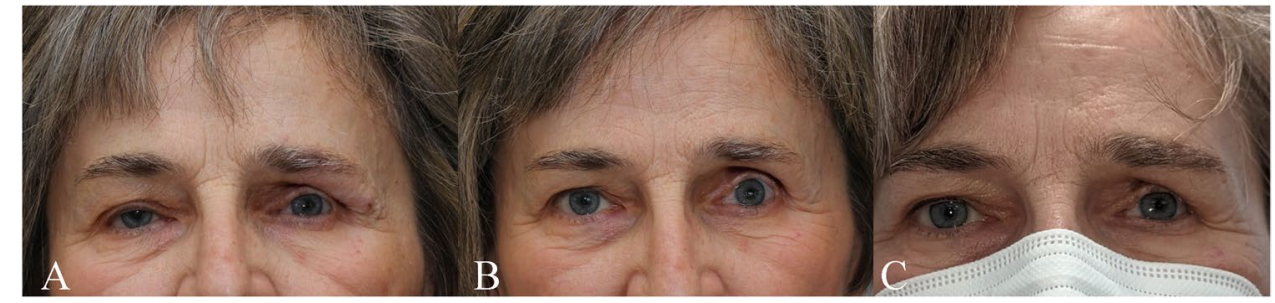


Fig. 2 Patient 1 closed eyelid view. Pre-treatment deficit (A) and 6-month follow-up (B)
Fig. 3 Patient 2 showing sovratarsal sulcus, tear trough, and scleral show (A). 1-year followup (B)
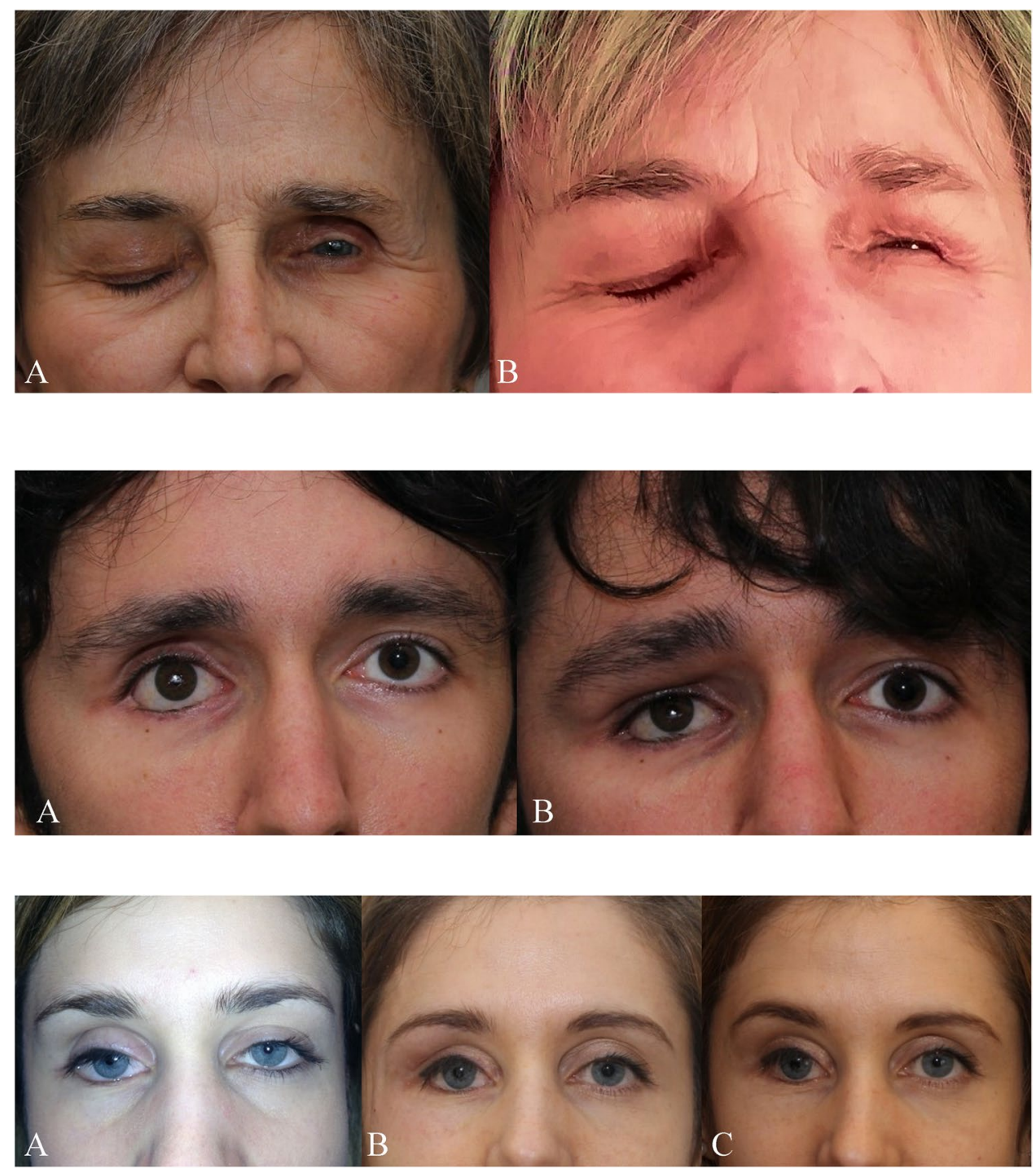

Fig. 4 Patient 3 open eyelid view. Pre-treatment (A), 3-year follow-up (B), and 6-year follow-up post touch up (C) contraction due to increased myofibroblasts activity, as seen in the early stages of wound healing following various injuries [1]. It is of paramount importance to surgically approach the anophthalmic cavity. Orbital implants of different materials, including porous, acrylic, and alloplastic non-porous spherical prosthesis, are used in socket rehabilitation.

The orbital volume deficiency is most often characterized by a sunken appearance of the ocular prosthesis and a deepened upper eyelid sulcus. In patients with PEESS whose orbital implant is either too small or surrounding fat atrophy is found, the therapeutic options include the substitution of the orbital implant, the placement of an orbital floor implant, dermis fat grafting, or autologous fat transfers [9-11]. An adjunctive orbital volume can be achieved with fat pearls, fat boluses, hydrogel implants, soft tissue grafting, and composite fillers.

The primary goal of anophthalmic socket reconstruction is to restore the anatomy fitting the appropriate prosthesis to achieve an aesthetically acceptable appearance. The reconstructive approach should adequately address the deficiencies in the orbital volume, the posterior lamella shrinkage, and the minified conjunctival surfaces. Although multiple surgical procedures are required to achieve a satisfactory result, socket rehabilitation becomes complex when the patient will not accept them due to complication risks like a retraction. In our experience, we encountered patients suffering from their aesthetic conditions, reporting a long history of surgeries and complications. Since these patients face an actual aesthetic problem despite the correct fit of the artificial eye, our treatment aims to avoid any retraction or complication that could cause the bad retention of the prosthesis. It is then mandatory to use a technique with no risk of complications and reversible if desired, such as HA injections.

The use of HA gel fillers has dramatically changed the approach in non-surgical rejuvenation and post-surgical 
corrections [12-18]. Since the rheological properties of different HA gel fillers may vary, the physician injects the gel to increase the projection, the volume or to define the anatomical areas choosing the appropriate product and face layers to inject, according to their specific features. In the present study, the filler with the higher G' gave better bony support, and more projection to the eyebrow, the one with lower G' was injected above the levator palpebrae superioris muscle to reduce the supra-tarsal sulcus, and to have a lower and better-defined skin crease. The ideal characteristics of softtissue fillers include acceptable longevity, biocompatibility, a low risk of migration, a minimal adverse event profile, and a reasonable cost-benefit ratio. For the aforementioned reasons, HA fillers are fit to produce volume enhancement in patients with upper eyelid sulcus, inferior tear trough deformity, and scleral show due to anophthalmic socket typical of PESS.

The use of filler based on calcium hydroxylapatite (CaHA) has been successfully reported for the orbital volume augmentation in craniofacial volume deficits [18-20]. It can be effective for orbital volume augmentation: the particles are biocompatible, resistant to phagocytosis, and migration through the lymphatic system.

Despite many advantages in anophthalmic orbital volume augmentation, calcium hydroxylapatite fillers are not completely risk-free from infections or extrusions, particularly when used in eyelids. Moreover, even if CaHA appears to be well-tolerated, there is no mechanism to dissolve the product in case of complications, undesirable results, or if it elicits a greater post-injection inflammatory response when compared to HA in terms of early local edema.

Fat grafting of the anophthalmic socket is a reconstructive technique extensively described in the literature [21-23]. As regards fat grafting in PESS, only few reports can be found [24-26]. Anderson et al. described Coleman's autologous fat grafting to the intraorbital, superior sulcus, and inferior sulcus region. The authors reported in their research a cosmetically unacceptable appearance due to inferior sulcus overfill. Such excess fat was removed 8-17 months following transplantation via a sub-ciliary or transconjunctival approach and if indicated, additional procedures such as tarsal strip and levator resection were performed at the same time. The authors conclude suggesting to inject fat only in the retroseptal orbital region via the superotemporal, inferotemporal, and inferior midline positions [24].

Although the fat grafting technique is frequently used in reconstructive surgery and in cosmetic volume augmentation, its results in PESS treatment may be inconsistent due to the hardly predictable fat resorption that vary from 40 to $60 \%$ of the injected volume [27].

Fillers used for facial rejuvenation need to fulfill important criteria: acceptable longevity, biocompatibility, minimal adverse reactions, and low risk of migration.
Therefore, HA injection is a minimally invasive technique that can be quickly performed in an outpatient setting and without surgical downtime. Moreover, HA fillers showed a semi-permanent volumizing effect in facial augmentations according to Mashiko [28]. In our research, we noticed a permanent effect, up to 8 years follow-up, in 11 out of 13 patients.

HA fillers offer a versatile and safe method for replacing soft tissue lost from the upper eyelid/brow complex in cases of PESS [29-31]. Unlike surgical approaches, they can be performed in an outpatient setting; furthermore, they have no associated donor site morbidity and no risk of graft hypertrophy, and they can be molded, with the potential for complete reversibility and minimal risk of lumpiness, bruising, or infection. If patients are disappointed with the results or complications to occur, HA can be dissolved easily and quickly by hyaluronidase injections, giving an edge over other fillers [32-34]. Furthermore, many of the patients we treated for this condition underwent multiple surgical procedures in the past, and they might prefer a less invasive alternative.

Several authors have observed discomfort, vasovagal symptoms, and potential oculocardiac reflex symptoms following HA injection in anophthalmic socket [29]. Following orbital HA injection, it is most likely that transient bradycardia and vasovagal symptoms including nausea are due to the stretching of the extraocular muscles [29]. Since our injection was focused on the eyelid rather than the orbital cavity, we did not experience such reactions. Unlike Zamani et al. described in their work, no complications as the mild, self-resolving eyelid edema or orbital cellulitis were reported [35].

As regards the longevity of the results, Leyngold et al. reported the HA retention time in the superior sulcus as 18 months; Malhotra reported an enophthalmos reduction $>1 \mathrm{~mm}$ for every $1 \mathrm{~mL}$ of HA injected. The longevity reported in the literature appears to be significantly lower compared to our experience [29, 31].

The present study demonstrated the effective use of HA injection for anophthalmic patients complaining about fat atrophy and asymmetry in volume replacement, correcting the deep superior sulcus crease, the tear trough deformity, and inferior scleral show.. The provided follow-up of approximately 8 years is the longest in the literature up to date. While the longevity of HA injected in the orbit could be variable, the present case series demonstrates a persistent volume augmentation over time. No major complications were reported. One case of abnormal bulging over the supra-tarsal crease was reported due to a HA superficial injection, resolved with one session of hyaluronidase injection.

Our experience illustrates that the HA injection is a very good option to reduce upper eyelid sulcus, and 
inferior tear trough and scleral show in post-enucleation socket, with excellent long-lasting results, no downtime, and/or major complications.

\section{Conclusion}

The post-enucleation socket syndrome can be improved thanks to HA filler injections. The right choice in filler features and injection technique is mandatory to achieve satisfactory and long-lasting results.

Author contribution Conceptualization, A.M.; methodology, G.F.N.; investigation, F.C.; resources, N.Z.; writing-original draft preparation, R.M.; writing-review and editing, G.L.G.; project administration, R.R. All authors have read and agreed to the final version of the manuscript.

Funding Open access funding provided by Università degli Studi di Napoli Federico II within the CRUI-CARE Agreement.

Data availability Data are available upon request.

\section{Declarations}

Ethical approval All procedures performed in studies involving human participants were in accordance with the ethical standards of the institutional and/or national research committee and with the 1964 Helsinki Declaration and its later amendments or comparable ethical standards. For this retrospective study no ethical approval is required.

Consent to participate Informed consent was obtained from all individual participants included in the study.

Consent for publication Patients signed informed consent regarding publishing their data and photographs.

Conflict of interest Renata Migliardi, Alessandra Modugno, Fabrizio Chirico, Nicola Zerbinati, Giovanni Francesco Nicoletti, Giorgio Lo Giudice, and Raffaele Rauso declare no competing interests.

Open Access This article is licensed under a Creative Commons Attribution 4.0 International License, which permits use, sharing, adaptation, distribution and reproduction in any medium or format, as long as you give appropriate credit to the original author(s) and the source, provide a link to the Creative Commons licence, and indicate if changes were made. The images or other third party material in this article are included in the article's Creative Commons licence, unless indicated otherwise in a credit line to the material. If material is not included in the article's Creative Commons licence and your intended use is not permitted by statutory regulation or exceeds the permitted use, you will need to obtain permission directly from the copyright holder. To view a copy of this licence, visit http://creativecommons.org/licenses/by/4.0/.

\section{References}

1. Hamedani M, Pournaras JA, Goldblum D (2007) Diagnosis and management of enophthalmos. Surv Ophthalmol 52:457-473. https://doi.org/10.1016/j.survophthal.2007.06.009

2. Antia NH, Arora S (1984) "Malignant" contracture of the eye socket. Plast Reconstr Surg 74:292-294. https://doi.org/10. 1097/00006534-198408000-00023

3. Soll DB (1982) The anophthalmic socket. Ophthalmology 89:407-423. https://doi.org/10.1016/s0161-6420(82)34774-0

4. Kaltreider SA, Lucarelli MJ (2002) A simple algorithm for selection of implant size for enucleation and evisceration: a prospective study. Ophthalmic Plast Reconstr Surg 18:336-341. https://doi.org/10.1097/00002341-200209000-00004

5. Camezind P, Robert PY, Adenis JP (2001) Post-enucleation or evisceration socket syndrome. Oper Tech Oculoplastic Orbital Reconstr Surg 4:48-51. https://doi.org/10.1076/otoo.4.1.48. 8490

6. Handler LF, Heher KL, Katowitz JA (1994) Congenital and acquired anophthalmia. Curr Opin Ophthalmol 5:84-90

7. Salti G, Rauso R (2015) Facial rejuvenation with fillers: the dual plane technique. J Cutan Aesthet Surg 8:127-133. https://doi.org/ 10.4103/0974-2077.167264

8. Tay E, Olver J (2008) Intraorbital hyaluronic acid for enophthalmos. Ophthalmology 115(1101-1101):e1102. https://doi.org/10. 1016/j.ophtha.2008.02.015

9. Cahill KV, Burns JA (1989) Volume augmentation of the anophthalmic orbit with cross-linked collagen (Zyplast). Arch Ophthalmol 107:1684-1686. https://doi.org/10.1001/archopht.1989. 01070020762044

10. Hunter PD, Baker SS (1994) The treatment of enophthalmos by orbital injection of fat autograft. Arch Otolaryngol Head Neck Surg 120:835-839. https://doi.org/10.1001/archotol.1994.01880 320037009

11. Huang ZL, Ma L (2005) Restoration of enophthalmos in anophthalmic socket by HTR polymer. Ophthalmic Plast Reconstr Surg 21:318-321. https://doi.org/10.1097/01.iop.0000170410. 23157.0e

12. Rauso R, Colella G, Zerbinati N, Salti G (2017) Safety and early satisfaction assessment of patients seeking nonsurgical rhinoplasty with filler. J Cutan Aesthet Surg 10:207-214. https://doi. org/10.4103/JCAS.JCAS_89_17

13. Rauso R, Tartaro G, Chirico F, Zerbinati N, Albani G, Rugge L (2020) Rhinofilling with hyaluronic acid thought as a cartilage graft. J Craniomaxillofac Surg 48:223-228. https://doi.org/10. 1016/j.jcms.2020.01.008

14. Rauso R, Federico F, Zerbinati N, De Cicco D, Nicoletti GF, Tartaro G (2020) Hyaluronic acid injections to correct lips deformity following surgical removal of permanent implant. J Craniofac Surg 31:e604-e606. https://doi.org/10.1097/SCS. 0000000000006689

15. Rauso R, Lo Giudice G, Zerbinati N, Tartaro G (2019) Hyaluronic acid injections as nonsurgical alternative in case of delayed diagnosis of malar arch fracture: case report and literature review. Case Rep Surg 2019:1360741. https://doi.org/10. 1155/2019/1360741

16. Monticelli D, Martina V, Mocchi R, Rauso R, Zerbinati U, Cipolla G, Zerbinati N (2019) Chemical characterization of hydrogels crosslinked with polyethylene glycol for soft tissue augmentation. Open Access Maced J Med Sci 7:1077-1081. https://doi.org/10.3889/oamjms.2019.279

17. Zerbinati N, Lotti T, Monticelli D, Rauso R, Gonzalez-Isaza P, D'Este E, Calligaro A, Sommatis S, Maccario C, Mocchi $R$ et al (2018) In vitro evaluation of the biosafety of hyaluronic acid PEG cross-linked with micromolecules of calcium 
hydroxyapatite in low concentration. Open Access Maced J Med Sci 6:15-19. https://doi.org/10.3889/oamjms.2018.044

18. Kotlus BS, Dryden RM (2007) Correction of anophthalmic enophthalmos with injectable calcium hydroxylapatite (Radiesse). Ophthalmic Plast Reconstr Surg 23:313-314. https://doi.org/10.1097/IOP.0b013e318068742c

19. Renno RZ (2007) Injectable calcium hydroxyapatite filler for minimally invasive delayed treatment of traumatic enophthalmos. Arch Facial Plast Surg 9:62-63. https://doi.org/10.1001/ archfaci.9.1.62

20. Vagefi MR, McMullan TF, Burroughs JR, White GL Jr, McCann JD, Anderson RL (2007) Injectable calcium hydroxylapatite for orbital volume augmentation. Arch Facial Plast Surg 9:439-442. https://doi.org/10.1001/archfaci.9.6.439

21. Jovanovic N, Carniciu AL, Russell WW, Jarocki A, Kahana A (2020) Reconstruction of the orbit and anophthalmic socket using the dermis fat graft: a major review. Ophthalmic Plast Reconstr Surg 36:529-539. https://doi.org/10.1097/IOP.00000 00000001610

22. Kuzmanovic Elabjer B, Busic M, Miletic D, Bjelos M, Saric B, Bosnar D (2018) Single-stage orbital socket reconstruction using the oversized dermis fat graft and the $22 \mathrm{~mm}$ silicone orbital implant after an extended enucleation. Case Rep Ophthalmol Med 2018:8954193. https://doi.org/10.1155/2018/ 8954193

23. Medel R, Alonso T, Pelaez F, Vasquez L (2016) Periumbilical fat auto-graft associated to a porous orbital implant for socket reconstruction after enucleation. Orbit 35:181-186. https://doi. org/10.1080/01676830.2016.1176206

24. Anderson OA, Tumuluri K, Francis ND, Olver JM (2008) Periocular autologous Coleman fat graft survival and histopathology. Ophthalmic Plast Reconstr Surg 24:213-217. https://doi.org/10. 1097/IOP.0b013e3181710321

25. Wilde F, Krauss O, Sakkas A, Mascha F, Pietzka S, Schramm A (2019) Custom wave-shaped CAD/CAM orbital wall implants for the management of post-enucleation socket syndrome. J Craniomaxillofac Surg 47:1398-1405. https://doi.org/10.1016/j.jcms. 2019.06.015

26. Aggarwal H, Singh K, Kumar P, Alvi HA (2013) A multidisciplinary approach for management of postenucleation socket syndrome with dermis-fat graft and ocular prosthesis: a clinical report. J Prosthodont 22:657-660. https://doi.org/10.1111/jopr. 12051

27. ELFadl D, Garimella V, Mahapatra TK, McManus PL, Drew PJ (2010) Lipomodelling of the breast: a review. Breast 19:202-209. https://doi.org/10.1016/j.breast.2010.02.009
28. Mashiko T, Mori H, Kato H, Doi K, Kuno S, Kinoshita K, Kunimatsu A, Ohtomo K Yoshimura, K (2013) Semipermanent volumization by an absorbable filler: onlay injection technique to the bone. Plast Reconstr Surg Glob Open:1(1):e4-e14 https://doi.org/ 10.1097/GOX.0b013e31828c66b0

29. Malhotra R (2007) Deep orbital Sub-Q restylane (nonanimal stabilized hyaluronic acid) for orbital volume enhancement in sighted and anophthalmic orbits. Arch Ophthalmol 125:1623-1629. https://doi.org/10.1001/archopht.125.12.1623

30. Feldman I, Sheptulin VA, Grusha YO, Malhotra R (2018) Deep orbital sub-Q hyaluronic acid filler injection for enophthalmic sighted eyes in Parry-Romberg syndrome. Ophthalmic Plast Reconstr Surg 34:449-451. https://doi.org/10.1097/IOP.00000 00000001050

31. Leyngold IM, Berbos ZJ, McCann JD, Pariseau B, Leyngold AR, Anderson RL (2014) Use of hyaluronic acid gel in the treatment of lagophthalmos in sunken superior sulcus syndrome. Ophthalmic Plast Reconstr Surg 30:175-179. https://doi.org/10.1097/IOP. 0000000000000040

32. Rauso R, Colella G, Franco R, Chirico F, Ronchi A, Federico F, Volpicelli A, Tartaro G (2019) Is hyaluronidase able to reverse embolism associated with hyaluronic acid filler? An anatomical case study. J Biol Regul Homeost Agents 33:1927-1930. https:// doi.org/10.23812/19-397-L

33. Rauso R, Zerbinati N, Franco R, Chirico F, Ronchi A, Sesenna E, Colella G, Tartaro G (2020) Cross-linked hyaluronic acid filler hydrolysis with hyaluronidase: different settings to reproduce different clinical scenarios. Dermatol Ther 33:e13269. https://doi. org/10.1111/dth.13269

34. Rauso R, Zerbinati N, Fragola R, Nicoletti GF, Tartaro G (2020) Transvascular hydrolysis of hyaluronic acid filler with hyaluronidase: an ex vivo study. Dermatol Surg. https://doi.org/10.1097/ DSS.0000000000002773,10.1097/DSS.0000000000002773

35. Zamani M, Thyagarajan S, Olver JM (2010) Adjunctive use of hyaluronic acid gel (Restylane Sub-Q) in anophthalmic volume deficient sockets and phthisical eyes. Ophthalmic Plast Reconstr Surg 26:250-253. https://doi.org/10.1097/IOP.0b013e3181ba76f5

Publisher's note Springer Nature remains neutral with regard to jurisdictional claims in published maps and institutional affiliations. 